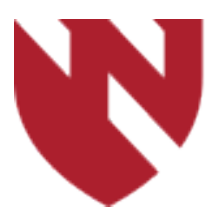

September 2020

\title{
Rare Case of Traumatic Tricuspid Valve Injury in Patient With Sinus Venosus Atrial Septal Defect (ASD)
}

\author{
Rishi Batra \\ University of Nebraska Medical Center \\ Laura Newton \\ University of Nebraska Medical Center \\ Nicholas Markin \\ University of Nebraska Medical Center \\ Samuel Cemaj \\ University of Nebraska Medical Center \\ Zachary Bauman \\ University of Nebraska Medical Center
}

Tell us how you used this information in this short survey.

Follow this and additional works at: https://digitalcommons.unmc.edu/gmerj

Part of the Higher Education Commons, and the Medicine and Health Sciences Commons

\section{Recommended Citation}

Batra, R., Newton, L., Markin, N., Cemaj, S., , Bauman, Z. Rare Case of Traumatic Tricuspid Valve Injury in Patient With Sinus Venosus Atrial Septal Defect (ASD). Graduate Medical Education Research Journal. 2020 Sep 29; 2(1).

https://digitalcommons.unmc.edu/gmerj/vol2/iss1/38

This Conference Proceeding is brought to you for free and open access by DigitalCommons@UNMC. It has been accepted for inclusion in Graduate Medical Education Research Journal by an authorized editor of DigitalCommons@UNMC.For more information, please contact digitalcommons@unmc.edu. 


\section{Rare Case of Traumatic Tricuspid Valve Injury in Patient With Sinus Venosus Atrial Septal Defect (ASD)}

\section{Creative Commons License}

\section{c) (1)@ $\Theta$}

This work is licensed under a Creative Commons Attribution-Noncommercial-No Derivative Works 4.0 License. 
Conclusion: Perioperative TXA administration was associated with reduced mean operative time and decreased intraoperative estimated blood loss. While not statistically significant, these results may be clinically significant. Larger, higher level studies are required for further investigation.

https://doi.org/10.32873/unmc.dc.gmerj.2.1.035

\section{Does Immediate Postoperative Opioid Consumption Correlate With Long-Term Outcomes in Patients Undergoing One and Two Level Instrumented Posterior Lumbar Fusions?}

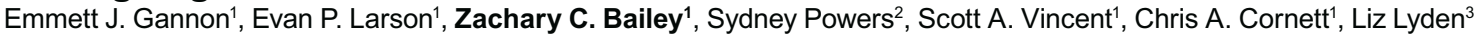

${ }^{1}$ University of Nebraska Medical Center, Department of Orthopaedic Surgery and Rehabilitation

${ }^{2}$ University of Nebraska Medical Center, College of Medicine

${ }^{3}$ University of Nebraska Medical Center, College of Public Health, Department of Biostatistics

Mentor: Chris Cornett

Program: Orthopaedic Surgery and Rehabilitation

Type: Original Research

Background: Opioid analgesics are routinely used following spine surgery. Preoperative and chronic postoperative use are associated with worse postoperative pain control and functional outcomes. Little is known about the effects of immediate postoperative use on long term outcomes. The purpose of this study is to investigate the potential relationship between increased opioid use immediately postoperatively and patient outcomes.
Methods: A retrospective review of patients undergoing instrumented 1 or 2-level posterior lumbar fusion surgery by a single surgeon was performed. Pearson correlation coefficients were used to evaluate associations between patient outcomes and immediate postoperative opioid use. Exclusion criteria included previous lumbar surgery, indications other than degenerative, and patients $<19$ years old. A total of 152 patients were included.

Results: Among 94 patients undergoing 1-level fusion, there was a significant inverse relationship between six month Short Form36 Bodily Pain and postoperative day (POD) 1,2 and total opioid use $(\mathrm{p}=0.0328, \mathrm{p}=0.0283$, and $\mathrm{p}=0.0205$ ). A significant inverse relationship was also found between 1 year Short Form-36 Physical Function and POD 1 and 2 opioid use $(\mathrm{p}=0.0415, \mathrm{p}=0.0200)$ and between 1 year Short Form-36 Bodily Pain and POD 2 opioid use ( $\mathrm{p}=0.0099)$. No correlation existed between amount of opioids consumed and outcomes after 2-level posterior lumbar fusion procedures.

Conclusion: The amount of opioid analgesics consumed in the immediate postoperative period may be helpful in predicting patient outcomes at 6 months and 1 year following 1-level posterior lumbar fusion. These findings, however, must be interpreted in context with the limitations inherent to a retrospective study.

https://doi.org/10.32873/unmc.dc.gmerj.2.1.036

\section{Rare Case of Traumatic Tricuspid Valve Injury in Patient with Sinus Venosus Atrial Septal Defect (ASD)}

Rishi Batra', Laura Newton ${ }^{1}$, Nicholas Markin², Samuel Cemaj', Zachary Bauman ${ }^{1}$

${ }^{1}$ University of Nebraska Medical Center, Department of Surgery

${ }^{2}$ University of Nebraska Medical Center, Department of Anesthesiology

Mentor: Zach Bauman

Program: General Surgery

Type: Case Report

Background: A 69-year-old male with history of congenital ASD repair as a child, atrial fibrillation and left ventricular dysfunction, presented as the restrained passenger of a motor vehicle crash. Initially complaining of left-sided chest pain, he was found to have left rib fractures 5-8 with flail physiology as well as a left tibial plateau and ulnar fracture. Due to flail chest physiology, open reduction and internal fixation (ORIF) of the ribs was scheduled.

Methods: After transthoracic echocardiogram (TTE) was completed, he was taken for ORIF. Upon intubation, he became acutely hypoxemic. Immediate left chest tube was placed, however, his clinical status remained unchanged. Emergent bronchoscopy was performed without resolution of the hypoxemia. Intraoperative transesophageal
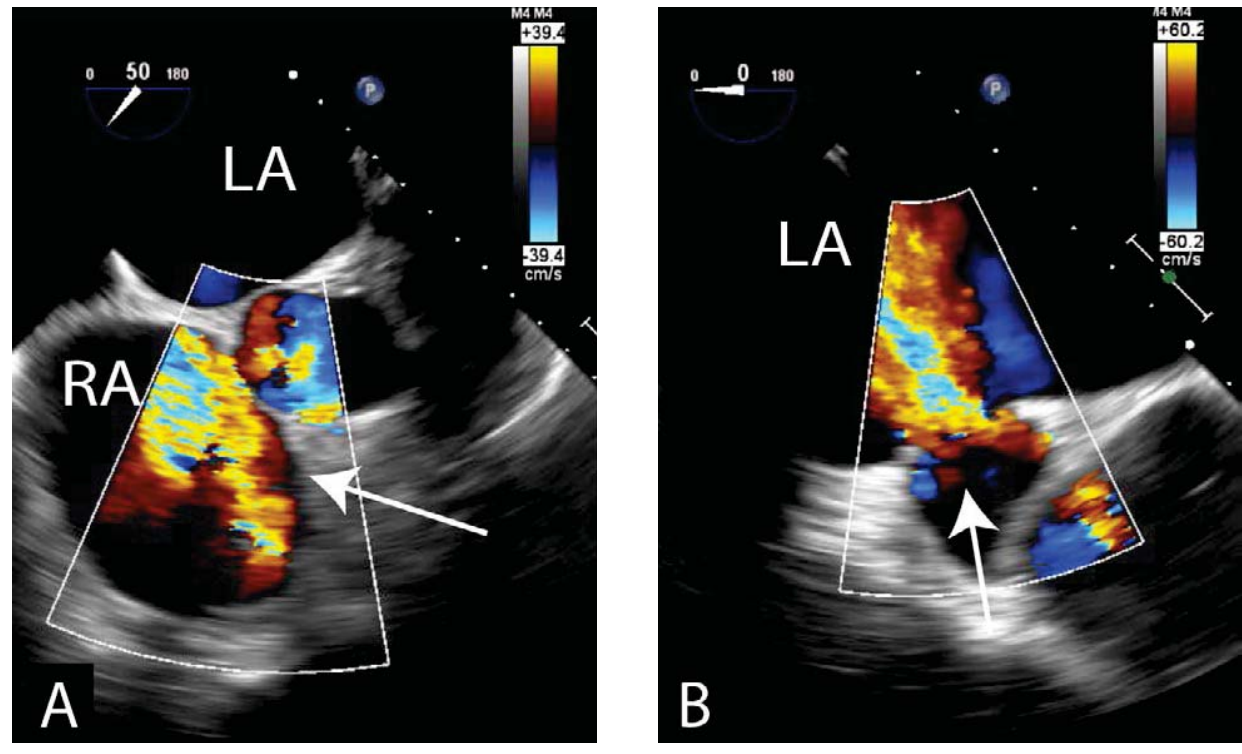

Figure 1A. Transesophageal echocardiogram showing the midesophageal modified right ventricular inflow view. There is severe tricuspid regurgitation (marked with arrow).

Figure 1B. Transesophageal echocardiogram showing a lower esophageal view of the inferior sinus venosus atrial septal defect. This image shows a high-velocity jet with right-to-left shunting. This is the result of the traumatic severe TR and the jet is directed at the sinus venosus atrial septal defect causing the right-to-left shunting (measuring $75 \mathrm{~mm} 2 \&<10 \mathrm{~mm} 2$ ). 
echocardiogram (TEE) was performed, revealing severe tricuspid regurgitation (TR) and significant right-to-left shunting. The procedure was subsequently aborted and epoprostenol initiated.

Results: A new flailed anterior leaflet of tricuspid valve (TV) was recognized during the attempted surgery, directing regurgitant blood flow through the residual sinus venosus ASD resulting in the right-to-left shunt. Unfortunately, this was never detected on
TTE. He remained in the ICU and developed impressive JVD. Repeat TEE confirmed traumatically ruptured anterior leaflet of the TV with severe TR worsened by the ASD. Overall, this resulted in depressed ejection fraction and a patient unfit for surgical repair of his injuries. Subsequently, he was taken to the OR by cardiac surgery for TV and sinus venous repair.

Conclusion: We present a patient with traumatic TR and history of congenital ASD.
Only $10 \%$ of cases of severe TR are primary due to processes directly affecting the TV. It is a rare traumatic injury usually resulting from blunt deceleration mechanisms. In fact, its prevalence may be underestimated due to subtle clinical manifestations. Therefore, it is imperative to maintain a high suspicion for such injuries, particularly in patients with a congenital cardiac history.

https://doi.org/10.32873/unmc.dc.gmerj.2.1.037

\section{You Can't Just Rub Some Dirt on It}

Alison K Bauer ${ }^{1}$, Lucas Bohannan', Jason Meredith ${ }^{1}$

${ }^{1}$ University of Nebraska Medical Center, Department of Family Medicine

\section{Mentor: Jason Meredith}

Program: Family Medicine

Type: Case Report

Introduction: Our case report demonstrates a classic presentation of compartment syndrome in an uncommon location.

Case: A 17 year-old male presented to a rural Nebraska clinic the day after sustaining a blunt trauma injury to his left anterior thigh during a high school football game. He was unable to finish the game secondary to pain. His pain increased throughout the night, leading to his presentation to clinic first thing the next morning. On exam, he was noted to have a firm, tender anterior thigh with extreme pain on passive range of motion testing. Additionally, decreased popliteal and dorsalis pedis pulses were found. An urgent CT scan revealed a vastus intermedius rupture, developing hematoma, and early compartment syndrome of the thigh. He was transferred by helicopter to a higher level of care for emergent fasciotomy. He subsequently underwent 5 procedures; ultimately our patient made a full recovery.

Discussion: This case illustrates the importance of early recognition and treatment of a potentially life-threatening orthopedic emergency despite its atypical location of presentation. Compartment syndrome of the thigh is rare compared to compartment syndrome of the lower leg and forearm. It is a clinical diagnosis requiring a high index of suspicion. Compartment pressure measurements, while available in some locations, is invasive, painful, and with questionable reliability. Early recognition and treatment of our patient's quad contusions at the time of his initial injury could have potentially prevented progression to a surgical emergency. In this case, quick and accurate recognition and evaluation led to expedited definitive, limb saving management.

https://doi.org/10.32873/unmc.dc.gmerj.2.1.038

\section{Dobhoff Tube Placement by ENT: Annoyance vs. Life Saving} Jenna Berg ${ }^{1}$, Christie Barnes ${ }^{1}$, Jayme Dowdall ${ }^{1}$

${ }^{1}$ University of Nebraska Medical Center, Department of Otolaryngology

Mentor: Jayme Dowdall

Program: Otolaryngology

Type: Original Research

Background: Dobhoff tube (DHT) placement seems like a simple procedure, but there can be serious associated morbidity and mortality including inadvertent placement of the tube into the lungs or through the skull base into the brain. ENT physicians can decrease this risk by placing the DHT through the nose under direct camera guidance in patients with elevated risk factors such as skull base fractures, prior skull base surgery, or high bleeding risk from low platelets or anticoagulation status. Placement of Dobhoff tubes in patients that do not meet these criteria consumes valuable time and resources as it requires an ENT resident to be present with a rigid nasal endoscope.
Methods: The number of DHT consults placed each month was recorded and the reason for each consult was analyzed to determine if it met criteria for a high risk patient. Each request for a DHT placement was recorded as a separate consult.

Results: There were a total of 20 DHT consults on 11 unique patients placed over a five-month period. Only three patients met criteria for being "high risk" with skull base fractures. five patients required multiple DHT placement, secondary to patient removal of the tube.

Conclusion: Less than $30 \%$ of the DHT consults in a five-month period were placed for high risk patients. This data supports revision of the current protocol to allow ENT review of the consult, and deferment to the hospital's specialized DHT placement team if the patient does not meet high risk criteria. 